that no compressed air is entering the tank and accordingly that the air valve is closed. Upon opening the inlet valve controlling admission of liquor through the feed opening, liquor is admitted. If this valve be closed when a proper charge has entered the tank and the air valve opened, conditions will be right for a discharge of the liquid contents. The discharge valve is opened, and this passage of liquid takes place. In the automatic monte-jus, the opening and closing of valves takes place without attention.

Monte-jus may be made of special chemical stoneware or of metal. In the latter case, they may be specially lined with lead, or the metal itself may be selected so as to provide suitable resistance to corrosion. A monte-jus is especially adapted for use with acids and liquors containing acids. It may be a small affair or it may be of considerable size.

Monte-jus of ten fit into special conditions. In one instance a filter press had to be fed with a thick granular and acid mixture. No pump could be found for the job. Gravity feed was unsuitable because of the probability that the connecting pipe line would suffer clogging. A monte-jus with a lead lining gave good service. Monte-jus are not cheap, either at the beginning or during operation. The first cost is considerable, and compressed air must be applied to secure action. They are not largely used in the United States but have found considerable application abroad. One great objection to the apparatus is its intermittent action. Under the special conditions where a gravity feed to the filter is permissible, however, the monte-jus may be used to supply the elevated tank without anything but inconsiderable fluctuations in the pressure in the press, provided the plan section of the tank is given a generous area. A monte-jus that is to be used under considerable air pressure may of ten with advantage be imbedded in concrete.

\section{Filter Aids}

\section{By C. P. Derleth}

Celite Products Co., Monadnock Bidg., Chicago, Ill.

A filter aid is defined as a substance which may be admixed 'with liquids to be filtered without affecting the physical or chemical characteristics of the filtrate and which facilitates the removal of suspended matter in the filtering process. Some of the materials which have been used for this purpose are: Asbestos fiber, wood pulp, paper pulp, Filter-Cel, diatomaceous earth and infusorial earth (both known as kieselguhr), magnesia, talc, lime, calcium sulfate, powdered silica, sawdust, and, partly for this purpose as well as for their decolorizing properties, vegetable carbon, animal carbons, and fuller's earth.

The material trade-named "Filter-Cel" is a cellular siliceous product which is especially prepared for filtration purposes, and of exceptional purity. Kieselguhr, which includes both diatomaceous earth and infusorial earth, is a broad term covering material from various deposits found throughout the world. The impurities occurring in these earths affect the filtering efficiency and contaminate the liquid with which they are admixed. Strata of clay are found in most deposits and, since they consist largely of alumina, have the effect of giving gelatinous precipitates, especially in alkaline solutions, which decrease the rate of filtration. Many of these clays are in such a colloidal state that the filtering efficiency of the material is completely counteracted.

Filtration problems may be divided into three classes:

1-Those in which the suspended solids are rigid, and either crystalline or amorphous.

2-Those in which the suspended solids are nonrigid bodies, i. e., those approaching the colloidal state, although they may be far greater in size than those properly classed as such.

3 -Suspensions containing mixtures of the above classes.
Fituration of Rigid Particles

Problems of the first class are the easiest to handle. It is necessary merely to choose a proper filtering medium, either cloth or screen. Usually the medium has a sufficiently fine mesh to retain all of the suspended solids from the beginning of the filtration period, but at times it is advantageous to use a medium with larger openings. In this case the suspended solids are depended on to build up, and the cloth or screen becomes, more properly speaking, the retaining medium. The filtration proper starts only when a properly arranged layer of larger sized particles has been retained, preventing the finer particles from passing through and giving true filtration.

In many problems in filtration the suspended solids have been produced by precipitation and crystallization. It is an advantage to secure the largest possible crystals, in such cases, so as to secure a cake having larger pores, thus offering less resistance to the flow of liquid.

In a case of this kind, provided the cake is not to be used, or if it can be subsequently separated from the filter aid, a precoating of the cloths with material of very fine porosity will give initial clarity. According to Hatschek, ${ }^{2}$ if the mean distance from pore to pore of the filtering medium is larger than the diameter of the particles being filtered, the first layer will be drawn by the vortices into the orifices of the pores and the medium throttled. It can be seen that if an initial film of very porous material be deposited on the medium the orifices will not be throttled. The network of pores prevents the meshes from being closed up, and each succeeding layer of deposit will form a network so that the whole thickness of the layer or film will be permeable. In addition it should always be kept in mind that it is advisable to work with reduced pressure at the beginning and gradually to increase the pressure as the thickness of the deposit increases. By this means the first layers of the deposit are spongy and porous, and offer little friction to the passage through of liquid, and the high final pressure tends to dry the cake. This precaution will also increase the length of life of the cloths by preventing the sharp corners of the crystals from cutting the fibers of the filter cloths, as a result of the pump pressure applied. Where corrosive liquid is being filtered the precoating of the cloths will give cleavage, allowing the cake to drop off readily, and will prevent the finer crystals from being forced into the pores of the retaining cloth or screen. Where fine mesh screens or metallic cloth are used, a precoating with a filter aid is often desirable to give initial clarity to the filtrate. The precoating may be done by charging the filtering surface with a suspension of the filter aid in water or clarified liquid, returning the filtrate by by-passing for a second filtration. It is easily possible with a good filter aid to retain finely divided crystals, such as barium sulfate.

\section{Filmation of Nonrigid Particles}

In a few instances precipitates of the second class can be converted into a crystalloidal state either by proper regulation of the precipitation or by subsequent treatment. This should be done wherever possible, thus converting the problem into one of the first class.

Those nonrigid suspended solids which do not lend themselves to crystallization and which have many of the properties and characteristics of colloids (álthough they may be much larger in size) constitute the most difficult problems in filtration.

A problem is often rendered more complex by the viscosity of the liquid, as in the case of concentrated sirups or raw linseed oil. In the past, gravity filtration has been depended upon in coping with this type of problem. The downward

1 J. Soc. Chem. Ind. 27 (1908), 539. 
movement of the liquid toward the filtering medium is so slow that the nonrigid solids settle down until they rest on the surface of the medium without being swept into its pores. As long as they are undisturbed they retain the shapes of the floc. For this reason a centrifugal pump is better adapted for pressure filtration than a reciprocal type, the pulsation of which causes a disturbance of the floc. Even an attempt to speed up the process by increasing the head results in driving the solids through and into the pores of the medium, yielding a cloudy filtrate. If the pores of the medium are fine enough to retain the solids, the filter is plugged and the operation is at an end.

Often the nonrigid solids are coagulated by heating or other means, or, if the solids do not lend themselves to coagulation, reagents have been added for the purpose of producing a flocculent precipitate and entangling the fine matter in suspension. The coagulant then collects on the surface of the filtering medium and a small head allows the liquid to trickle through slowly. The disadvantage of this method is the slowness of the operation, necessitating a very large filtering area.

USE OF FILTER AID-If the somewhat uniformly sized particles of a filter aid are added to the liquid containing the nonrigid particles, the gelatinous particles are separated upon being deposited on the filtering medium, layer after layer being built. The porous particles give rigidity to the cake, permitting pressure to be used and preventing the particles from being swept through the pores of the retaining medium, thus making it possible to secure perfect clarity. The pores in the filter aid should be microscopic, the filtration being accomplished more through capillaries than between interspaces of the particles, inasmuch as the interspaces are filled with the albuminous, pectinous, colloidal, or other solid matter.

The volume of filter aid necessary will depend upon the quantity of suspended matter present, the temperature at which filtration is carried on, and the pressure used, as well as upon the viscosity and specific gravity of liquid being filtered, etc., and this quantity can best be determined experimentally, by using an excess of the filter aid, and gradually decreasing the amount added until the rate of flow of the filtrate is retarded, indicating that the surface pores are becoming clogged.

\section{Necessary Characteristics of a Filter Aid}

POROSITY-Porosity is a criterion of filtering efficiency. Crystalline bodies such as magnesia and calcium sulfate occupy definite spaces which do not completely cover the surface of the filter, but pack adjacent to each other's edges and surfaces, and have small or large channels according to their shape and size, which being rigid are not affected by pressure and which offer free passage to the filtrate. Particles of a material such as Filter-Cel pack adjacent to each other or adjacent to the particles of suspended matter in the liquid, but because they are porous they can retain relatively larger quantities of precipitate, at the same time maintaining a continuity of pores through the cake to the retaining medium.

REGULARITY OF sIZE-Particles should be as regular in size as possible. Small particles will pack in the interspaces left by the larger grains, reducing the size of the free passages, and causing the rate per square foot of filtering area to be retarded.

INERT MATERIAL-The product should be practically inert. If there is an appreciable soluble matter which would affect the taste, odor, color, or chemical analysis, or in any other way reduce the purity of the liquid to be filtered it will not be effective. Care should be taken in the selection of a filter aid to suit the liquid handled. Hot caustic liquors dissolve silica readily. Magnesia, used on a small scale in pharmaceutical work, is soluble in every acid liquor.

BULKINESS-A filter aid should be light in weight and bulky. It is purchased by weight and used by bulk, therefore its weight per cubic foot in the dry and loose form is an important item. It is important not only from the cost standpoint, but also in the manipulation of the liquor in the filtration process. The particles should settle out so slowly that excessive settling will not take place while the mixture of the liquor with the filter aid is in the filtering equipment. If the material were to settle out rapidly it would not build up an even, porous cake, but would build up solidly from the bottom, and that portion of the cake space filled or bridged would be ineffective for the filtering operation. When the filter becomes about half full of solid cake the rate is so retarded that it is usually not possible to wait until the entire cake space is filled. In the case of a leaf type filter, the heavy material would settle out in the bottom of the shell and seriously affect the rate.

A filter aid should be added to build up an open, porous cake which will fill, so far as possible, the cake space in the filter. This makes possible thorough washing or blowing of the cake to reduce retention of liquid, permits the filter to be cleaned readily, and allows the filter cloths to retain their porosity for longer lengths of time. There is probably an absorption of colloidal matter by the filter aid, as is shown by the removal to a trace of the albumin of milk by a simple filtration with Filter-Cel.

\section{Advantages of Using a Filter Aid}

1-Improved clarity of filtrate.

2 -Saving in power due to reduction of pressure.

3 -Less loss of liquid by cake retention, because a firm dry cake is built.

4-Increased extraction through ability to wash cake.

5-Less labor in cleaning cloths, also less redressing.

6-Decreased cloth expense.

7-Increased rates resulting in greater capacity of filtering equipment.

8-Makes possible the use of labor-saving types of pressure filters for clarification filtrations.

9 -Complete separation of solids from liquids without the use of chemical defecants, and without affecting chemical composition of the filtrate.

\section{The Use of Filter-Cel for Industrial Filtration Processes}

\author{
By G. M. Hickey
}

Celite Products Co., NEW YorK, N. Y.

In every filtering operation in which the filtrate is of value, and in cases where the cake is dissolved, Filter-Cel can be used to an advantage. In order to show the wide use of Filter-Cel to filtering operations, its application in four typical industries is outlined: the manufacture of cereal beverages; the refining of coconut oil; the hydrogenation of oil and production of glycerol from soap manufacturing; and the production of cider and allied products from the pressed fruit juice.

\section{Cereal Beverage Filtration}

The former method of manufacturing cereal beverages involved long settling processes, after which the beverage was easily clarified with a pulp filter. The present low alcoholic content of cereal beverages presents a different problem and two new methods are used: the checked fermentation and the dealcoholization process. In either one there is a large quantity of suspended solids that must be removed. Some manufacturers mix Filter-Cel with their pulp to give a finer 